\title{
Direct and Practical Synthesis of 2-Arylbenzoxazoles Promoted by Activated Carbon
}

\author{
Yuka Kawashita, Natsuki Nakamichi, Hirotoshi Kawabata, and \\ Masahiko Hayashi*
}

Department of Chemistry, Faculty of Science, Kobe University, Nada, Kobe, 657-8501, Japan

mhayashi@kobe-u.ac.jp

\section{Supporting Information}

General methods. All melting points were measured on a Yanaco MP-500D and uncorrected. ${ }^{1} \mathrm{H}$ and ${ }^{13} \mathrm{C}$ NMR spectra (400 and 100.6 MHz, respectively) were recorded on a JEOL JNM-LA 400 using $\mathrm{Me}_{4} \mathrm{Si}$ as the internal standard. IR spectra were measured with a PERKIN ELMER FT-IR Spectrometer SPECTRUM 1000. Elemental analyses were performed with a Yanaco CHN Corder MT-5. Mass spectra were taken on a Shimadzu GCMS-QP 2000A. Preparative column chromatography was carried out on Fuji Silysia BW-820MH. Thin-layer chromatography (TLC) was carried out on foil plates, Silica Gel $60 \mathrm{~F}_{254}$ (E. Merck; layer thickness $\left.0.2 \mathrm{~mm}\right)$.

Typical procedure is as follows (entry 1 in Table 2): A mixture of 2-aminophenol (873 mg, 8 mmol), benzaldehyde ( 849 $\mathrm{mg}, 8 \mathrm{mmol})$ and $\operatorname{Darco}^{\circledR} \mathrm{KB}(1 \mathrm{~g})$ in xylene $(15 \mathrm{~mL})$ was placed in a $100 \mathrm{~mL}$ three-necked flask under an oxygen atmosphere and stirred at $120{ }^{\circ} \mathrm{C}$ for $4 \mathrm{~h}$. The reaction mixture was then filtered using Celite. After the filtrate was concentrated, the product was isolated by silica gel column chromatography to give a pale yellow crystalline solid $1.2 \mathrm{~g}$ $\left(78 \%\right.$ ). When $0.2 \mathrm{~g}$ of activated carbon (Darco ${ }^{\circledR} \mathrm{KB}$ ) was used in the above reaction (in the same substrate scale), a longer reaction time was required $(18 \mathrm{~h}, 85 \%)$.

2-phenylbenzoxazole (entry 1 in Table 2)

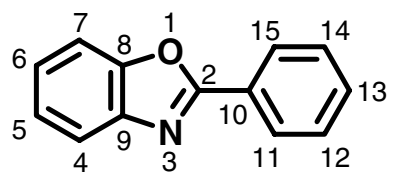

$R_{f} 0.28$ (30:1 hexane ${ }^{-}$ethyl acetate); mp $106^{-} 107^{\circ} \mathrm{C}$ (lit. $\left.{ }^{6} 102{ }^{\circ} \mathrm{C}\right)$; IR (KBr): $v_{\max }\left(\mathrm{cm}^{-1}\right) 3060,1617,1553,1490,1479,1472$,

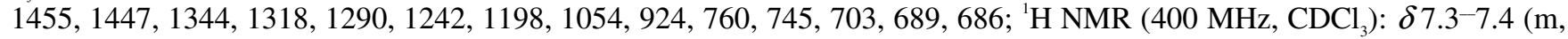
$\left.2 \mathrm{H}, \mathrm{H}_{6}, \mathrm{H}_{5}\right), 7.5^{-7.6}\left(\mathrm{~m}, 3 \mathrm{H}, \mathrm{H}_{12}, \mathrm{H}_{13}, \mathrm{H}_{14}\right), 7.5^{-7} .6\left(\mathrm{~m}, 1 \mathrm{H}, \mathrm{H}_{7}\right), 7^{-7} 7.8\left(\mathrm{~m}, 1 \mathrm{H}, \mathrm{H}_{4}\right), 8.2^{-} 8.3\left(\mathrm{~m}, 2 \mathrm{H}, \mathrm{H}_{11}, \mathrm{H}_{15}\right) ;{ }^{13} \mathrm{C} \mathrm{NMR}$ $\left(100.6 \mathrm{MHz}, \mathrm{CDCl}_{3}\right): \delta 110.5(\mathrm{C}-7), 120.0(\mathrm{C}-4), 124.5(\mathrm{C}-6), 125.1(\mathrm{C}-5), 127.2(\mathrm{C}-13), 127.6(\mathrm{C}-11, \mathrm{C}-15), 128.9(\mathrm{C}-12$, C-14), 131.5 (C-10), 142.1 (C-9), 150.7 (C-8), 163.0 (C-2); MS m/z (relative intensity): 195 (100\%), 167 (37.7\%), 77 (32.9\%), 64 (47.8\%), 63 (76.1\%), 51 (26.2\%); Anal. Calcd for $\mathrm{C}_{13} \mathrm{H}_{9} \mathrm{NO}$ : C, 79.98; H, 4.65; N, 7.17. Found: C, 79.98; H, $4.66 ; \mathrm{N}, 7.19$.

2-(4'-methylphenyl)benzoxazole (entry 2 in Table 2)

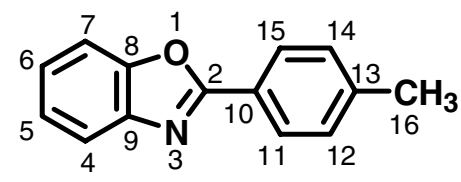




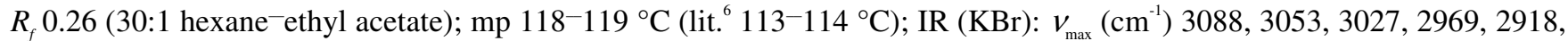

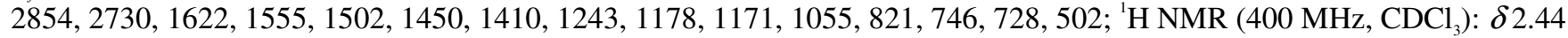
$\left(\mathrm{s}, 3 \mathrm{H}, \mathrm{H}_{16}\right), 7.3-7.4\left(\mathrm{~m}, 4 \mathrm{H}, \mathrm{H}_{6}, \mathrm{H}_{5}, \mathrm{H}_{12}, \mathrm{H}_{14}\right), 7.5^{-7.6}\left(\mathrm{~m}, 1 \mathrm{H}, \mathrm{H}_{7}\right), 7.7-7.8\left(\mathrm{~m}, 1 \mathrm{H}, \mathrm{H}_{4}\right), 8.15\left(\mathrm{~d}, 2 \mathrm{H}, J_{\text {H11-Н12 }}=J_{\text {H15-H14 }}=8.0 \mathrm{~Hz}\right.$, $\left.\mathrm{H}_{11}, \mathrm{H}_{15}\right) ;{ }^{13} \mathrm{C}$ NMR (100.6 MHz, CDCl $): \delta 21.6(\mathrm{C}-16), 110.5$ (C-7), 119.8 (C-4), $124.4(\mathrm{C}-6), 124.5(\mathrm{C}-5), 124.8(\mathrm{C}-10)$, 127.6 (C-11, C-15), 129.6 (C-12, C-14), 142.0 (C-9), 142.2 (C-13), 150.7 (C-8), 163.3 (C-2); MS m/z (relative intensity): 210 (23.8\%), 209 (100\%), 208 (36.6\%), 91 (30.9\%), 64 (37.5\%), 63 (63.7\%); Anal. Calcd for $\mathrm{C}_{14} \mathrm{H}_{11} \mathrm{NO}$ : C, 80.36; H, 5.31; N, 6.67. Found: C, 80.39; H, 5.31; N, 6.67.

2-(4'-methoxyphenyl)benzoxazole (2)

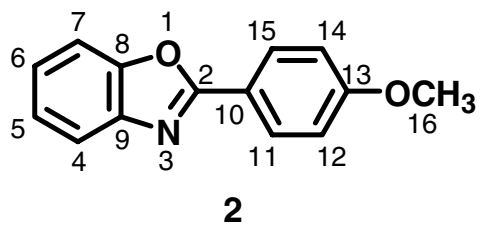

$R_{f} 0.28$ (10:1 hexane-ethyl acetate); mp 101- $103{ }^{\circ} \mathrm{C}$ (lit. $\left.{ }^{6} 101{ }^{\circ} \mathrm{C}\right)$; IR $(\mathrm{KBr}): v_{\max }\left(\mathrm{cm}^{-1}\right) 3051,3021,2958,2930,2910,2835$, $1921,1887,1619,1605,1505,1454,1255,1185,1170,1025,786,743,730,524 ;{ }^{1} \mathrm{H} \mathrm{NMR}\left(400 \mathrm{MHz}, \mathrm{CDCl}_{3}\right): \delta 3.89(\mathrm{~s}$, $\left.3 \mathrm{H}, \mathrm{H}_{16}\right), 7.03\left(\mathrm{ddd}, 2 \mathrm{H}, J_{\mathrm{H} 12-\mathrm{H} 11}=J_{\mathrm{H} 14 \mathrm{H} 15}=9.5 \mathrm{~Hz}, J_{\mathrm{H} 12-\mathrm{H} 14}=2.5 \mathrm{~Hz}, J_{\mathrm{H} 12-\mathrm{H} 15}=J_{\mathrm{H} 14-\mathrm{H} 11}=2.5 \mathrm{~Hz}, \mathrm{H}_{12}, \mathrm{H}_{14}\right), 7.3^{-7.4}\left(\mathrm{~m}, 2 \mathrm{H}, \mathrm{H}_{6}, \mathrm{H}_{5}\right)$, 7.5-7.6 (m, $\left.1 \mathrm{H}, \mathrm{H}_{7}\right), 7.7^{-7.8}\left(\mathrm{~m}, 1 \mathrm{H}, \mathrm{H}_{4}\right), 8.20\left(\mathrm{ddd}, 2 \mathrm{H}, J_{\mathrm{H} 1-\mathrm{H} 12}=J_{\mathrm{H} 15-\mathrm{H} 14}=9.5 \mathrm{~Hz}, J_{\mathrm{H} 1-\mathrm{H} 15}=2.5 \mathrm{~Hz}, J_{\mathrm{H} 1-\mathrm{H} 14}=J_{\mathrm{H} 15-\mathrm{H} 12}=2.5 \mathrm{~Hz}\right.$, $\left.\mathrm{H}_{11}, \mathrm{H}_{15}\right) ;{ }^{13} \mathrm{C} \mathrm{NMR}\left(100.6 \mathrm{MHz}, \mathrm{CDCl}_{3}\right): \delta 55.4(\mathrm{C}-16), 110.4(\mathrm{C}-7), 114.4(\mathrm{C}-12, \mathrm{C}-14), 119.6(\mathrm{C}-4), 119.7(\mathrm{C}-10), 124.4$ (C-6), 124.6 (C-5), 129.4 (C-11, C-15), 142.3 (C-9), 150.7 (C-8), 162.3 (C-13), 163.2 (C-2); MS m/z (relative intensity): $226(16.4 \%), 225$ (100\%), 210 (36.6\%), 182 (35.3\%), 64 (16.6\%), 63 (26.7\%); Anal. Calcd for $\mathrm{C}_{14} \mathrm{H}_{11} \mathrm{NO}_{2}: \mathrm{C}, 74.65 ; \mathrm{H}$, 4.92; N, 6.22. Found: C, 74.64; H, 4.85; N, 6.22.

2-(4'-chlorophenyl)benzoxazole (entry 4 in Table 2)

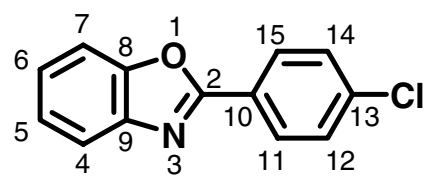

$R_{f} 0.34$ (30:1 hexane-ethyl acetate); $\mathrm{mp} 153^{-} 154^{\circ} \mathrm{C}$ (lit..$\left.^{6} 147^{\circ} \mathrm{C}\right) ; \mathrm{IR}(\mathrm{KBr}): v_{\max }\left(\mathrm{cm}^{-1}\right) 3088,3074,3058,3022,1617,1484$, $1477,1472,1452,1405,1343,1245,1092,1057,1052,1012,832,758,739,498 ;{ }^{1} \mathrm{H}$ NMR $\left(400 \mathrm{MHz}, \mathrm{CDCl}_{3}\right): \delta 7.3^{-7} .4$ $\left(\mathrm{m}, 2 \mathrm{H}, \mathrm{H}_{6}, \mathrm{H}_{5}\right), 7.51\left(\mathrm{ddd}, 2 \mathrm{H}, J_{\mathrm{H} 1-\mathrm{H} 12}=J_{\mathrm{H} 15-\mathrm{H} 14}=9.1 \mathrm{~Hz}, J_{\mathrm{H} 11-\mathrm{H} 15}=2.2 \mathrm{~Hz}, J_{\mathrm{H} 11-\mathrm{H} 14}=J_{\mathrm{H} 15-\mathrm{H} 12}=2.2 \mathrm{~Hz}, \mathrm{H}_{11}, \mathrm{H}_{15}\right), 7.5-7.6(\mathrm{~m}, 1 \mathrm{H}$, $\left.\mathrm{H}_{7}\right), 7.7-7.8\left(\mathrm{~m}, 1 \mathrm{H}, \mathrm{H}_{4}\right), 8.20\left(\mathrm{ddd}, 2 \mathrm{H}, J_{\mathrm{H} 12-\mathrm{H} 11}=J_{\mathrm{H} 14-\mathrm{H} 15}=9.1 \mathrm{~Hz}, J_{\mathrm{H} 12-\mathrm{H} 14}=2.2 \mathrm{~Hz}, J_{\mathrm{H} 12-\mathrm{H} 15}=J_{\mathrm{H} 14-\mathrm{H} 11}=2.2 \mathrm{~Hz}, \mathrm{H}_{12}, \mathrm{H}_{14}\right) ;{ }^{13} \mathrm{C} \mathrm{NMR}$ $\left(100.6 \mathrm{MHz}, \mathrm{CDCl}_{3}\right): \delta 110.6(\mathrm{C}-7), 120.1(\mathrm{C}-4), 124.7$ (C-6), $125.3(\mathrm{C}-5), 125.7(\mathrm{C}-10), 128.8$ (C-11, C-15), $129.2(\mathrm{C}-12$, C-14), 137.7 (C-13), 142.0 (C-9), 150.8 (C-8), 162.0 (C-2); MS m/z (relative intensity): 231 (33.1\%), 229 (100\%), 92 (17.6\%), 64 (49.7\%), 63 (62.1\%), 38 (19.2\%); Anal. Calcd for $\mathrm{C}_{13} \mathrm{H}_{8} \mathrm{ClNO}$ : C, 67.99; H, 3.51; N, 6.10. Found: C, 68.15; H, $3.55 ; \mathrm{N}, 6.05$.

2-(4'-cyanophenyl)benzoxazole (entry 5 in Table 2)

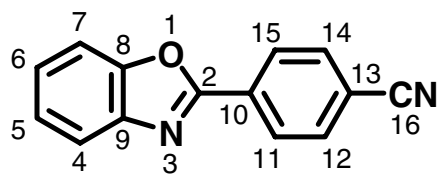

$R_{f} 0.27$ (10:1 hexane-ethyl acetate); $\mathrm{mp} 207-209^{\circ} \mathrm{C}$ (lit. $\left.{ }^{9} 207-208^{\circ} \mathrm{C}\right) ; \mathrm{IR}(\mathrm{KBr}): v_{\max }\left(\mathrm{cm}^{-1}\right) 3095,3051,3047,2229,1600$, $1494,1475,1452,1411,1343,1242,1056,1013,843,817,761,751,693,622,548 ;{ }^{1}{ }^{H} \mathrm{NMR}^{2}\left(400 \mathrm{MHz}, \mathrm{CDCl}_{3}\right): \delta 7.4^{-7} 7.6$ $\left(\mathrm{m}, 2 \mathrm{H}, \mathrm{H}_{6}, \mathrm{H}_{5}\right), 7.6^{-7.7}\left(\mathrm{~m}, 1 \mathrm{H}, \mathrm{H}_{7}\right), 7.8^{-7.9}\left(\mathrm{~m}, 3 \mathrm{H}, \mathrm{H}_{4}, \mathrm{H}_{12}, \mathrm{H}_{14}\right), 8.37\left(\mathrm{ddd}, 2 \mathrm{H}, J_{\mathrm{H} 1-\mathrm{H} 12}=J_{\mathrm{H} 15-\mathrm{H} 14}=8.6 \mathrm{~Hz}, J_{\mathrm{H} 11-\mathrm{H} 15}=1.7 \mathrm{~Hz}\right.$, $\left.J_{\mathrm{H} 1-\mathrm{H} 14}=J_{\mathrm{H} 15-\mathrm{H} 12}=1.7 \mathrm{~Hz}, \mathrm{H}_{11}, \mathrm{H}_{15}\right) ;{ }^{13} \mathrm{C}$ NMR $\left(100.6 \mathrm{MHz}, \mathrm{CDCl}_{3}\right): \delta 110.8(\mathrm{C}-7), 114.8(\mathrm{C}-13), 118.1(\mathrm{C}-16), 120.6(\mathrm{C}-4)$, 125.1 (C-6), 126.1 (C-5), 127.9 (C-11, C-15), 131.1 (C-10), 132.7 (C-12, C-14), 141.9 (C-9), 150.9 (C-8), 160.9 (C-2); MS $\mathrm{m} / z$ (relative intensity): 221 (15.9\%), 220 (100\%), 192 (12.4\%), 64 (31.1\%), 63 (36.3\%), 38 (10.2\%); Anal. Calcd for $\mathrm{C}_{14} \mathrm{H}_{8} \mathrm{~N}_{2} \mathrm{O}: \mathrm{C}, 76.35 ; \mathrm{H}, 3.66 ; \mathrm{N}, 12.72$. Found: C, 76.45; H, 3.78; N, 12.55 . 


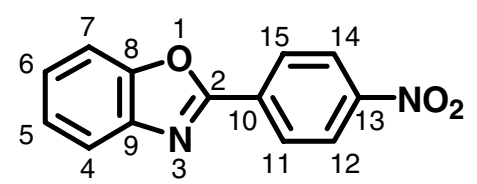

$R_{f} 0.29$ (15:1 hexane-ethyl acetate); mp 269-270 ${ }^{\circ} \mathrm{C}$ (lit. $\left.{ }^{6} 266^{-} 268^{\circ} \mathrm{C}\right) ; \mathrm{IR}(\mathrm{KBr}): v_{\max }\left(\mathrm{cm}^{-1}\right) 3107,3093,3056,3049,2845$, $1597,1554,1521,1451,1347,1338,1301,1240,1105,1058,857,852,766,753,707 ;{ }^{1} \mathrm{H}$ NMR $\left(400 \mathrm{MHz}, \mathrm{CDCl}_{3}\right): \delta$ $7.4-7.5\left(\mathrm{~m}, 2 \mathrm{H}, \mathrm{H}_{6}, \mathrm{H}_{5}\right), 7.64\left(\mathrm{dd}, 1 \mathrm{H}, J_{\mathrm{H} 7-\mathrm{H} 6}=6.5 \mathrm{~Hz}, J_{\mathrm{H} 7-\mathrm{H} 5}=2.7 \mathrm{~Hz}, \mathrm{H}_{7}\right), 7.83\left(\mathrm{dd}, 1 \mathrm{H}, J_{\mathrm{H} 4-\mathrm{H} 5}=6.5 \mathrm{~Hz}, J_{\mathrm{H} 4-\mathrm{H} 6}=2.7 \mathrm{~Hz}, \mathrm{H}_{4}\right)$, 8.4-8.5 (m, 4H, $\left.\mathrm{H}_{11}, \mathrm{H}_{12}, \mathrm{H}_{14}, \mathrm{H}_{15}\right) ;{ }^{13} \mathrm{C}$ NMR (100.6 MHz, CDCl $)$ : $\delta 111.0(\mathrm{C}-7), 120.7(\mathrm{C}-4), 124.2(\mathrm{C}-11, \mathrm{C}-15), 125.2(\mathrm{C}-$ 6), 126.3 (C-5), 128.4 (C-12, C-14), 132.8 (C-10), 141.9 (C-9), 149.4 (C-13), 151.0 (C-8), 160.7 (C-2); MS m/z (relative intensity): 240 (100\%), 210 (20.6\%), 194 (31.6\%), 182 (20.6\%), 139 (20.9\%), 64 (22.4\%), 63 (38.1\%); Anal. Calcd for $\mathrm{C}_{13} \mathrm{H}_{8} \mathrm{~N}_{2} \mathrm{O}_{3}: \mathrm{C}, 65.00 ; \mathrm{H}, 3.36 ; \mathrm{N}, 11.66$. Found: $\mathrm{C}, 64.94 ; \mathrm{H}, 3.32 ; \mathrm{N}, 11.53$.

5-methyl-2-phenylbenzoxazole (entry 7 in Table 2)

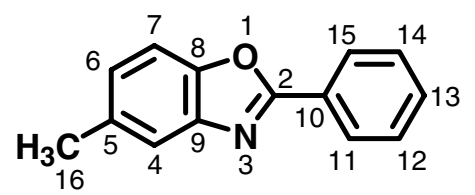

$R_{f} 0.26$ (30:1 hexane ${ }^{-}$ethyl acetate); $\mathrm{mp} 108{ }^{\circ} \mathrm{C}\left(\right.$ lit. $\left.^{6} 103{ }^{\circ} \mathrm{C}\right)$; IR $(\mathrm{KBr}): v_{\max }\left(\mathrm{cm}^{-1}\right) 3056,2918,2863,1956,1894,1818$, $1766,1725,1584,1552,1475,1447,1295,1199,1021,927,827,797,701,597 ;{ }^{1} \mathrm{H} \mathrm{NMR}\left(400 \mathrm{MHz}, \mathrm{CDCl}_{3}\right): \delta 2.48(\mathrm{~s}, 3 \mathrm{H}$, $\left.\mathrm{H}_{16}\right), 7.15\left(\mathrm{dd}, 1 \mathrm{H}, J_{\mathrm{H} 6-\mathrm{H} 7}=8.3 \mathrm{~Hz}, J_{\mathrm{H} 6-\mathrm{H} 4}=1.2 \mathrm{~Hz}, \mathrm{H}_{6}\right), 7.44\left(\mathrm{~d}, 1 \mathrm{H}, J_{\mathrm{H} 7 \mathrm{-H} 6}=8.3 \mathrm{~Hz}, \mathrm{H}_{7}\right), 7.4-7.5\left(\mathrm{~m}, 3 \mathrm{H}, \mathrm{H}_{12}, \mathrm{H}_{13}, \mathrm{H}_{14}\right), 7.55(\mathrm{~d}$, $\left.1 \mathrm{H}, J_{\mathrm{H} 4-\mathrm{H} 6}=1.2 \mathrm{~Hz}, \mathrm{H}_{4}\right), 8.2-8.3\left(\mathrm{~m}, 2 \mathrm{H}, \mathrm{H}_{11}, \mathrm{H}_{15}\right) ;{ }^{13} \mathrm{C}$ NMR $\left(100.6 \mathrm{MHz}, \mathrm{CDCl}_{3}\right): \delta 21.4(\mathrm{C}-16), 109.8(\mathrm{C}-7), 119.9(\mathrm{C}-4)$, 126.1 (C-6), 127.3 (C-10), 127.5 (C-11, C-15), 128.8 (C-12, C-14), 131.2 (C-13), 134.3 (C-5), 142.3 (C-9), 148.9 (C-8), 163.0 (C-2); MS m/z (relative intensity): 209 (100\%), 208 (33.6\%), 78 (48.0\%), 77 (41.5\%), 52 (25.7\%), 51 (37.3\%); Anal. Calcd for $\mathrm{C}_{14} \mathrm{H}_{11} \mathrm{NO}$ : C, 80.36; H, 5.30; N, 6.69. Found: C, 80.34; H, 5.37; N, 6.73.

5-nitro-2-phenylbenzoxazole (entry 8 in Table 2)

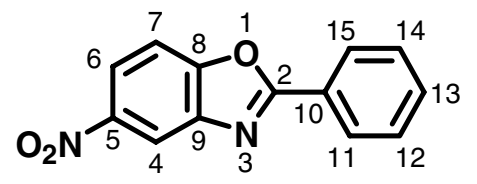

$R_{f} 0.26$ (15:1 hexane-ethyl acetate); $\mathrm{mp} 177^{\circ} \mathrm{C}$ (lit. $\left.{ }^{9} 171.5^{-172.5}{ }^{\circ} \mathrm{C}\right) ; \mathrm{IR}(\mathrm{KBr}): v_{\max }\left(\mathrm{cm}^{-1}\right) 3100,3070,3042,2861,1910$, $1615,1553,1527,1482,1450,1350,1338,1320,1267,1024,821,738,705,689,489 ;{ }^{1} \mathrm{H}$ NMR (400 MHz, $\left.\mathrm{CDCl}_{3}\right): \delta$ 7.5-7.6 (m, 3H, $\left.\mathrm{H}_{12}, \mathrm{H}_{13}, \mathrm{H}_{14}\right), 7.69\left(\mathrm{~d}, 1 \mathrm{H}, J_{\mathrm{H} 7 \mathrm{H} 6}=9.2 \mathrm{~Hz}, \mathrm{H}_{7}\right), 8.2-8.3\left(\mathrm{~m}, 2 \mathrm{H}, \mathrm{H}_{11}, \mathrm{H}_{15}\right), 8.33\left(\mathrm{dd}, 1 \mathrm{H}, J_{\mathrm{H} 6-\mathrm{H} 7}=9.2 \mathrm{~Hz}, J_{\mathrm{H} 6 \mathrm{H} 4}=\right.$ $\left.2.0 \mathrm{~Hz}, \mathrm{H}_{6}\right), 8.66\left(\mathrm{~d}, 1 \mathrm{H}, J_{\mathrm{H}-\mathrm{H} 6}=2.0 \mathrm{~Hz}, \mathrm{H}_{4}\right) ;{ }^{13} \mathrm{C} \mathrm{NMR}\left(100.6 \mathrm{MHz}, \mathrm{CDCl}_{3}\right): \delta 110.7(\mathrm{C}-7), 116.2(\mathrm{C}-4), 121.1(\mathrm{C}-6), 125.9$ (C-10), 128.0 (C-11, C-15), 129.1 (C-12, C-14), 132.6 (C-13), 142.5 (C-9), 145.4 (C-5), 154.2 (C-8), 165.9 (C-2); MS m/z (relative intensity): 240 (100\%), 194 (52.9\%), 91 (100\%), 63 (100\%), 62 (41.1\%), 51 (43.5\%); Anal. Calcd for $\mathrm{C}_{13} \mathrm{H}_{8} \mathrm{~N}_{2} \mathrm{O}_{3}$ : C, 65.00; H, 3.36; N, 11.66. Found: C, 64.94; H, 3.42; N, 11.58

2-(4'-methoxyphenyl)5-nitrobenzoxazole (entry 9 in Table 2)

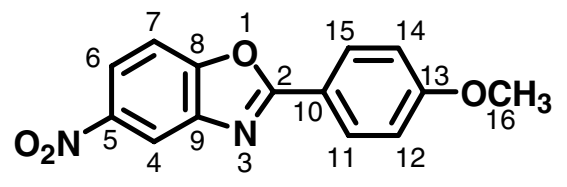

$R_{f} 0.21$ (10:1 hexane-ethyl acetate); mp $188^{\circ} \mathrm{C}$ (lit. $\left.{ }^{9} 184^{-} 186^{\circ} \mathrm{C}\right)$; IR $(\mathrm{KBr}): v_{\max }\left(\mathrm{cm}^{-1}\right) 3108,3066,3011,2969,2948,2917$, $2841,1616,1602,1551,1520,1426,1345,1252,1173,1022,839,822,745,521 ;{ }^{1} \mathrm{H} \mathrm{NMR}\left(400 \mathrm{MHz}, \mathrm{CDCl}_{3}\right): \delta 3.92(\mathrm{~s}$, $\left.3 \mathrm{H}, \mathrm{H}_{16}\right), 7.06\left(\mathrm{ddd}, 2 \mathrm{H}, J_{\mathrm{H} 12-\mathrm{H} 11}=J_{\mathrm{H} 14-\mathrm{H} 15}=9.5 \mathrm{~Hz}, J_{\mathrm{H} 12-\mathrm{H} 14}=2.5 \mathrm{~Hz}, J_{\mathrm{H} 12-\mathrm{H} 15}=J_{\mathrm{H} 14-\mathrm{H} 11}=2.5 \mathrm{~Hz}, \mathrm{H}_{12}, \mathrm{H}_{14}\right), 7.65\left(\mathrm{~d}, 1 \mathrm{H}, J_{\mathrm{H} 7-\mathrm{H} 6}=8.8\right.$ 
$\left.\mathrm{Hz}, \mathrm{H}_{7}\right), 8.23\left(\mathrm{ddd}, 2 \mathrm{H}, J_{\mathrm{H} 1-\mathrm{H} 12}=J_{\mathrm{H} 15-\mathrm{H} 14}=9.5 \mathrm{~Hz}, J_{\mathrm{H} 1-\mathrm{H} 15}=2.5 \mathrm{~Hz}, J_{\mathrm{H} 1-\mathrm{H} 14}=J_{\mathrm{H} 15-\mathrm{H} 12}=2.5 \mathrm{~Hz}, \mathrm{H}_{11}, \mathrm{H}_{15}\right), 8.29\left(\mathrm{dd}, 1 \mathrm{H}, J_{\mathrm{H} 6 \mathrm{H} \mathrm{H}}=8.8\right.$ $\left.\mathrm{Hz}, J_{\mathrm{H} 6-\mathrm{H} 4}=2.1 \mathrm{~Hz}, \mathrm{H}_{6}\right), 8.60\left(\mathrm{~d}, 1 \mathrm{H}, J_{\mathrm{H} 4-\mathrm{H} 6}=2.1 \mathrm{~Hz}, \mathrm{H}_{4}\right) ;{ }^{13} \mathrm{C} \mathrm{NMR}\left(100.6 \mathrm{MHz}, \mathrm{CDCl}_{3}\right): \delta 55.5(\mathrm{C}-16), 110.4(\mathrm{C}-7), 114.6$ (C-12, C-14), 115.7 (C-4), 118.3 (C-6), 120.6 (C-10), 129.9 (C-11, C-15), 142.8 (C-9), 145.3 (C-5), 154.3 (C-8), 163.2 (C13), 166.1 (C-2); MS m/z (relative intensity): 271 (15.7\%), 270 (100\%), 224 (49.4\%), 91 (58.6\%), 79 (13.6\%), 63 (59.3\%); Anal. Calcd for $\mathrm{C}_{14} \mathrm{H}_{10} \mathrm{~N}_{2} \mathrm{O}_{4}:$ C, 62.22; H, 3.73; N, 10.37. Found: C, 62.42; H, 3.77; N, 10.32 .

2-(4'-chlorophenyl)5-nitrobenzoxazole (entry 10 in Table 2)

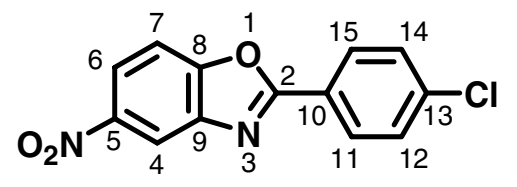

$R_{f} 0.28$ (15:1 hexane-ethyl acetate); mp $219^{\circ} \mathrm{C}$ (lit. $\left.{ }^{9} 218^{-} 219^{\circ} \mathrm{C}\right)$; IR $(\mathrm{KBr}): v_{\max }\left(\mathrm{cm}^{-1}\right) 3051,3021,2958,2930,2910,2835$, 1921, 1887, 1619, 1605, 1505, 1454, 1255, 1185, 1170, 1025, 786, 743, 730, 524; ${ }^{1} \mathrm{H}$ NMR (400 MHz, CDCl $): \delta 7.55$ (ddd, $\left.2 \mathrm{H}, J_{\mathrm{H} 12-\mathrm{H} 11}=J_{\mathrm{H} 14-\mathrm{H} 15}=9.1 \mathrm{~Hz}, J_{\mathrm{H} 12-\mathrm{H} 14}=2.3 \mathrm{~Hz}, J_{\mathrm{H} 14-\mathrm{H} 11}=J_{\mathrm{H} 12-\mathrm{H} 15}=2.3 \mathrm{~Hz}, \mathrm{H}_{12}, \mathrm{H}_{14}\right), 7.69\left(\mathrm{~d}, 1 \mathrm{H}, J_{\mathrm{H} 7-\mathrm{H} 6}=9.1 \mathrm{~Hz}, \mathrm{H}_{7}\right), 8.22$ (ddd, $\left.2 \mathrm{H}, J_{\mathrm{H} 1-\mathrm{H} 12}=J_{\mathrm{H} 15-\mathrm{H} 14}=9.1 \mathrm{~Hz}, J_{\mathrm{H} 1-\mathrm{H} 15}=2.3 \mathrm{~Hz}, J_{\mathrm{H} 1-\mathrm{H} 14}=J_{\mathrm{H} 15-\mathrm{H} 12}=2.3 \mathrm{~Hz}, \mathrm{H}_{11}, \mathrm{H}_{15}\right), 8.34\left(\mathrm{dd}, 1 \mathrm{H}, J_{\mathrm{H} 6-\mathrm{H} 7}=9.1 \mathrm{~Hz}, J_{\mathrm{H} 6 \mathrm{H} 4}=2.1 \mathrm{~Hz}\right.$, $\left.\mathrm{H}_{6}\right), 8.65\left(\mathrm{~d}, 1 \mathrm{H}, J_{\mathrm{H} 4 \mathrm{H} 6}=2.1 \mathrm{~Hz}, \mathrm{H}_{4}\right) ;{ }^{13} \mathrm{C}$ NMR $\left(100.6 \mathrm{MHz}, \mathrm{CDCl}_{3}\right): \delta 110.8(\mathrm{C}-7), 116.4(\mathrm{C}-4), 121.4(\mathrm{C}-6), 124.5(\mathrm{C}-10)$, 129.3 (C-11, C-15), 129.6 (C-12, C-14), 139.1 (C-13), 142.5 (C-9), 145.6 (C-5), 154.3 (C-8), 165.1 (C-2); MS m/z (relative intensity): 276 (28.1\%), 274 (83.5\%), 228 (30.4\%), 91 (100\%), 63 (78.5\%), 62 (24.3\%); Anal. Calcd for $\mathrm{C}_{13} \mathrm{H}_{7} \mathrm{ClN}_{2} \mathrm{O}_{3}: \mathrm{C}_{\text {, }}$ $56.85 ; \mathrm{H}, 2.57$; N, 10.20. Found: C, 57.04; H, 2.57; N, 10.22 .

2-pivalbenzoxazole (3)

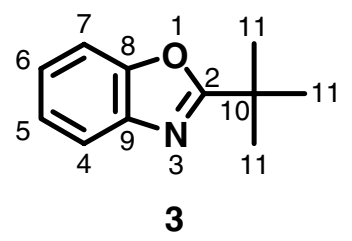

$R_{f} 0.31$ (60:1 hexane-ethyl acetate); IR (KBr): $v_{\max }\left(\mathrm{cm}^{-1}\right) 3057,2974,2934,2906,2871,1706,1611,1564,1456,1293$, $1244,1126,1099,1002,929,790,765,751,736,408 ;{ }^{1} \mathrm{H}$ NMR $\left(400 \mathrm{MHz}, \mathrm{CDCl}_{3}\right): \delta 1.50\left(\mathrm{~s}, 9 \mathrm{H}, \mathrm{H}_{11}\right), 7.2^{-} 7.3\left(\mathrm{~m}, 2 \mathrm{H}, \mathrm{H}_{6}\right.$, $\left.\mathrm{H}_{5}\right)$, 7.4-7.5 (m, $\left.1 \mathrm{H}, \mathrm{H}_{7}\right), 7.6^{-7.7}\left(\mathrm{~m}, 1 \mathrm{H}, \mathrm{H}_{4}\right) ;{ }^{13} \mathrm{C}$ NMR $\left(100.6 \mathrm{MHz}, \mathrm{CDCl}_{3}\right): \delta 28.5(\mathrm{C}-11), 34.2(\mathrm{C}-10), 110.3(\mathrm{C}-7)$, 119.7 (C-4), 123.9 (C-6), 124.4 (C-5), 141.3 (C-9), 150.8 (C-8), 173.5 (C-2); MS m/z (relative intensity): 175 (32.8\%), 160 (100\%), 133 (36.9\%), 119 (31.0\%), 41 (53.5\%), 39 (32.0\%); Anal. Calcd for $\mathrm{C}_{11} \mathrm{H}_{13} \mathrm{NO}: \mathrm{C}, 75.40 ; \mathrm{H}, 7.48$; N, 7.99. Found: C, 75.46; H, 7.54; N, 7.96.

2-cyclohexylbenzoxazole (4)

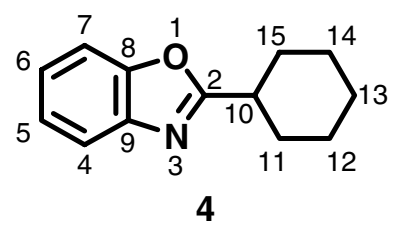

$R_{f} 0.22$ (50:1 hexane-ethyl acetate); $\mathrm{mp} 35^{\circ} \mathrm{C}$; IR $(\mathrm{KBr}): v_{\max }\left(\mathrm{cm}^{-1}\right) 3054,3019,2954,2922,2850,1611,1568,1460,1449$, 1376, 1245, 1160, 1130, 1017, 944, 893, 837, 757, 742, 425; ${ }^{1} \mathrm{H}$ NMR (400 MHz, $\left.\mathrm{CDCl}_{3}\right): \delta 1.3^{-1.5}\left(\mathrm{~m}, 3 \mathrm{H}, \mathrm{H}_{12}, \mathrm{H}_{13}, \mathrm{H}_{14}\right)$, 1.7-1.8 (m, 3H, $\left.\mathrm{H}_{11}, \mathrm{H}_{13}, \mathrm{H}_{15}\right), 1.8-1.9\left(\mathrm{~m}, 2 \mathrm{H}, \mathrm{H}_{12}, \mathrm{H}_{14}\right), 2.1^{-} 2.2\left(\mathrm{~m}, 2 \mathrm{H}, \mathrm{H}_{11}, \mathrm{H}_{15}\right), 2.96\left(\mathrm{tt}, 1 \mathrm{H}, J_{\mathrm{H} 10-\mathrm{H} 11}=J_{\mathrm{H} 10 \mathrm{H} 15^{\prime}}=11.3 \mathrm{~Hz}\right.$ $\left.J_{\mathrm{H} 10-\mathrm{H} 11}=J_{\mathrm{H} 10-\mathrm{H} 15}=3.7 \mathrm{~Hz}, \mathrm{H}_{10}\right), 7.2^{-7.3}\left(\mathrm{~m}, 2 \mathrm{H}, \mathrm{H}_{6}, \mathrm{H}_{5}\right), 7.4^{-7.5}\left(\mathrm{~m}, 1 \mathrm{H}, \mathrm{H}_{7}\right), 7.6^{-7.7}\left(\mathrm{~m}, 1 \mathrm{H}, \mathrm{H}_{4}\right) ;{ }^{13} \mathrm{C} \mathrm{NMR}(100.6 \mathrm{MHz}$, $\left.\mathrm{CDCl}_{3}\right): \delta 25.6(\mathrm{C}-12, \mathrm{C}-14), 25.8(\mathrm{C}-13), 30.5(\mathrm{C}-11, \mathrm{C}-15), 37.9(\mathrm{C}-10), 110.2(\mathrm{C}-7), 119.6(\mathrm{C}-4), 123.9(\mathrm{C}-6), 124.3(\mathrm{C}-$ 5), 141.3 (C-9), 150.6 (C-8), 170.4 (C-2); MS m/z (relative intensity): 201 (11.3\%), 172 (13.0\%), 160 (11.9\%), 147 (14.9\%), $146(88.3 \%), 133$ (100\%), 65 (11.3\%), 64 (13.6\%), 63 (16.7\%), 55 (17.6\%), 41 (32.0\%), 39 (21.2\%); Anal. Calcd for $\mathrm{C}_{13} \mathrm{H}_{15} \mathrm{NO}: \mathrm{C}, 77.58 ; \mathrm{H}, 7.51 ; \mathrm{N}, 6.96$. Found: $\mathrm{C}, 77.47 ; \mathrm{H}, 7.59 ; \mathrm{N}, 7.07$. 


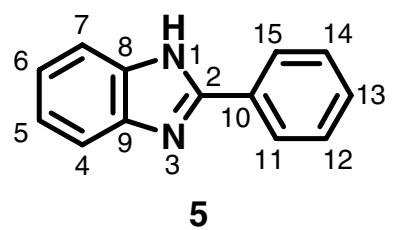

$R_{f} 0.36$ (30:1 hexane-ethyl acetate); mp 299-301 ${ }^{\circ} \mathrm{C}$ (lit. $\left.{ }^{8} 296^{-} 297.5^{\circ} \mathrm{C}\right) ; \mathrm{IR}(\mathrm{KBr}): \nu_{\max }\left(\mathrm{cm}^{-1}\right) 3046,2967,2834,2718$, $2675,2534,1890,1591,1463,1445,1411,1316,1277,1227,1120,745,738,704,687,561$; ${ }^{1} \mathrm{H}$ NMR (400 MHz, DMSO$\left.d_{6}\right): \delta 7.1^{-7.2}\left(\mathrm{~m}, 2 \mathrm{H}, \mathrm{H}_{6}, \mathrm{H}_{5}\right), 7.5^{-7} 7.6\left(\mathrm{~m}, 4 \mathrm{H}, \mathrm{H}_{7}, \mathrm{H}_{12}, \mathrm{H}_{13}, \mathrm{H}_{14}\right), 7.67\left(\mathrm{~d}, 1 \mathrm{H}, J_{\mathrm{H}-\mathrm{H} 5}=7.6 \mathrm{~Hz}, \mathrm{H}_{4}\right), 8.1^{-} 8.2\left(\mathrm{~m}, 2 \mathrm{H}, \mathrm{H}_{11}, \mathrm{H}_{15}\right)$, $12.9\left(\mathrm{~s}, 1 \mathrm{H}, \mathrm{H}_{1}\right) ;{ }^{13} \mathrm{C}$ NMR $\left(100.6 \mathrm{MHz}\right.$, DMSO- $\left.d_{6}\right): \delta 111.5(\mathrm{C}-7), 119.0(\mathrm{C}-4), 121.8(\mathrm{C}-6), 122.7(\mathrm{C}-5), 126.6(\mathrm{C}-12$, C14), 129.1 (C-11, C-15), 130.0 (C-13), 130.3 (C-10), 135.2 (C-9), 144.0 (C-8), 151.4 (C-2); MS m/z (relative intensity): 195 (13.9\%), 194 (100\%), 193 (26.0\%), 97 (13.5\%), 64 (16.0\%), 63 (18.2\%); Anal. Calcd for $\mathrm{C}_{13} \mathrm{H}_{10} \mathrm{~N}_{2}: \mathrm{C}, 80.39 ; \mathrm{H}, 5.19 ; \mathrm{N}$, 14.42. Found: C, 80.25; H, 5.20; N, 14.35 . 
Analytical data of ICP-AES

\begin{tabular}{|c|c|c|c|}
\hline Element & $\begin{array}{c}\operatorname{Darco}^{\circledR} \mathrm{KB} \\
\quad(\mathrm{ppm})\end{array}$ & $\begin{array}{c}\operatorname{Darco}^{\circledR} \mathrm{KB}-\mathrm{B} \\
(\mathrm{ppm})\end{array}$ & $\begin{array}{l}\text { Darco }{ }^{\circledR} \text { G-60 } \\
\quad(\mathrm{ppm})\end{array}$ \\
\hline $\mathrm{Li}$ & 34.5 & 41.5 & 0.32 \\
\hline B & 6.61 & 20 & 4.93 \\
\hline $\mathrm{Na}$ & 1750 & 1753 & 268 \\
\hline $\mathrm{Mg}$ & 86.9 & 54.80 & 409 \\
\hline $\mathrm{Al}$ & 831 & 1130 & 156 \\
\hline $\mathrm{Si}$ & 200 & 130 & 302 \\
\hline $\mathrm{P}$ & 3931 & 3710 & 197 \\
\hline $\mathrm{K}$ & 517 & 575 & 43.1 \\
\hline $\mathrm{Ca}$ & 605.5 & 423 & 544.0 \\
\hline $\mathrm{Ti}$ & 38.05 & 30.52 & 73.2 \\
\hline $\mathrm{Cr}$ & 6.9 & 5.80 & $-^{\mathrm{b}}$ \\
\hline $\mathrm{Mn}$ & 9.99 & 4.1 & 6.5 \\
\hline $\mathrm{Fe}$ & 299 & 98.3 & 175 \\
\hline $\mathrm{Cu}$ & 16.77 & 29.8 & 6.88 \\
\hline $\mathrm{Zn}$ & 5.36 & 31.8 & 37 \\
\hline $\mathrm{Sr}$ & 12.3 & 7.07 & 6.01 \\
\hline $\mathrm{Y}$ & 0.67 & $-^{\mathrm{a}}$ & $-^{b}$ \\
\hline $\mathrm{Sn}$ & 12 & 13 & $\_^{\mathrm{b}}$ \\
\hline $\mathrm{Ba}$ & 14.6 & 7.76 & 22.6 \\
\hline $\mathrm{Eu}$ & 0.23 & $-^{\mathrm{a}}$ & $-^{\mathrm{a}}$ \\
\hline Но & 0.45 & $-^{\mathrm{b}}$ & 0.6 \\
\hline $\mathrm{Er}$ & 9.33 & 7.83 & 18.3 \\
\hline $\mathrm{W}$ & 5.59 & 4.06 & $-^{\mathrm{b}}$ \\
\hline
\end{tabular}

$\operatorname{Darco}^{\circledR} \mathrm{KB}$ : The following elements were not observed above the level of determination limit (10 $\sigma$ method); $\mathrm{V}, \mathrm{Co}, \mathrm{Ni}, \mathrm{As}$, $\mathrm{Rb}, \mathrm{Mo}, \mathrm{Ru}, \mathrm{Pd}, \mathrm{In}, \mathrm{Nd}, \mathrm{Tb}$, Dy. The following elements were not observed above the level of detection limit ( $3 \sigma$ method); $\mathrm{Be}, \mathrm{Ga}, \mathrm{As}, \mathrm{Se}, \mathrm{Zr}, \mathrm{Rh}, \mathrm{Ag}, \mathrm{Cd}, \mathrm{Sb}, \mathrm{La}, \mathrm{Ce}, \mathrm{Sm}, \mathrm{Pr}, \mathrm{Gd}, \mathrm{Yb}, \mathrm{Re}, \mathrm{Os}, \mathrm{Ir}, \mathrm{Pt}, \mathrm{Au}, \mathrm{Pb}, \mathrm{Bi}$.

Darco ${ }^{\circledR}$ KB-B: The following elements were not observed above the level of determination limit (10 $\sigma$ method); Ni, Y, Zr, $\mathrm{Mo}, \mathrm{Ru}, \mathrm{Pd}, \mathrm{Ag}, \mathrm{In}, \mathrm{Sb}, \mathrm{Pr}, \mathrm{Nd}, \mathrm{Eu}, \mathrm{Tb}, \mathrm{Pt}, \mathrm{Pb}$. The following elements were not observed above the level of detection limit (3 $\sigma$ method); Be, V, Co, Ga, As, Se, Rb, Rh, Cd, Sb, La, Ce, Sm, Gd, Dy, Ho, Yb, Re, Os, Ir, Au, Bi.

$\operatorname{Darco}^{\circledR}$ G-60: The following elements were not observed above the level of determination limit; $\mathrm{Be}, \mathrm{Co}, \mathrm{Ni}, \mathrm{Se}, \mathrm{Zr}, \mathrm{Ru}, \mathrm{Pd}$, In, $\mathrm{Nd}, \mathrm{Eu}, \mathrm{Tb}, \mathrm{Pt}$ (10 $\sigma$ method). The following elements were not observed above the level of detection limit (3 $\sigma$ method); V, Cr, Co, Ga, As, Rb, Y, Mo, Rh, Ag, Cd, Sn, Sb, La, Ce, Pr, Sm, Gd, Dy, Yb, W, Re, Os, Ir, Au, Pb, Bi. 